\title{
Melioidosis: A Retrospective Review of Orthopaedic Manifestations
}

\author{
S Ahmad, MBBS, L Azura, MS Ortho, S Duski, MS Ortho, MY Aziz, MS Ortho \\ Department of Orthopaedic, Sultan Haji Ahmad Shah Hospital, Temerloh, Malaysia
}

\begin{abstract}
Melioidosis is an infectious disease which is associated with high rate of mortality. We reviewed 33 patients treated in our hospital over a period of 14 months. About half $(55 \%)$ of these patient presented with one focus of infection while the rest had multifocal infections. $81 \%$ require drainage of abscess or arthrotomy. Four patients (12\%) died as a result of septicaemia and the rate is lower than those reported in literature. We conclude that mortality of melioidosis can reduce with early diagnosis followed by appropriate and prolonged antibiotic therapy.
\end{abstract}

Key Words:

Melioidosis, Burkholderia pseudomallei

\section{INTRODUCTION}

Melioidosis is an infectious disease that is caused by Burkholderia pseudomallei first described in Rangoon, Burma in 1912 by Whitmore and Krishnaswami ${ }^{1}$, amongst debilitated homeless morphine addicts. Burkholderia pseudomallei is commonly found in the soil and water, and is endemic in Asia, Southeast Asia and Northern Australia ${ }^{2}$. Infection with this organism is associated with high mortality rate in animals and humans in the tropics ${ }^{3}$. Most infection was thought to be via percutaneous inoculation and can affect any part of the body ${ }^{4}$. It has a broad spectrum of clinical manifestations and can mimic other infections both clinically and radiologically ${ }^{5,6}$.

There is usually a multisystem involvement in Burkholderia pseudomallei infection. Pneumonia is the most common presentation and is associated with a high mortality rate $^{2,3,4,5,10,11}$. Extremity presentation of melioidosis is common in the orthopaedic setting and includes septic arthritis and infection involving skin and subcutaneous tissue ${ }^{3,19,20}$. Increased awareness and a high index of suspicion are required to reduce the morbidity and mortality due to this infection. We conducted this study to review melioidosis cases treated in this institution and their outcome of treatment.

\section{MATERIALS AND METHODS}

This is a cross-sectional retrospective study conducted in our institution. All medical records of confirmed melioidosis, with or without orthopaedic referral dating from December 2006 to January 2008, were reviewed and analysed in terms of demographic data, clinical signs, symptoms, clinical investigation and management.

All cases with positive cultures were treated accordingly. In cases with negative culture results, but with a high index of suspicion of melioidosis, serum for serology was sent to Kuala Lumpur Hospital for analysis. All patients with positive culture or serology were included in this study.

\section{RESULTS}

A total of 33 patients with confirmed melioidosis were treated in our hospital from December 2006 to January 2008. The male to female ratio is $8: 3$. Their age ranged from 40 to 65 years old. Eight patients were from the same district $(72 \%)$ while the rest were referred from the surrounding districts. There was no specific occupation correlated with the infection; however all three female patients were housewives. Nine patients were diabetics while the other two had no co-morbid illness. The patients presented to the hospital with various complaints including fever, swelling, purulent discharge and other symptoms of sepsis.

The majority of patients (55\%) had only a single focus of infection, $18 \%$ had two organs/areas involvement and $27 \%$ had sepsis involving 3 organs/areas. Four out of 33 patients died within a few days of admission due to overwhelming sepsis. Most septicaemia was related to pneumonia. Abscesses were the most common form of infection involving $63.6 \%$ of patients, followed by septic arthritis $(27 \%)$; the remaining patients had cellulitis with septicaemia. The majority $(81 \%)$ of patients required surgical intervention such as drainage and arthrotomy. The majority of the diagnoses were confirmed from tissue or blood cultures. Only two had negative cultures and were diagnosed solely based on serology. 
The patients were all started on antibiotics from the first day of admission. The initial choice of antibiotics depended on whether the patient had sepsis or just localized infection. Seven patients were started with ceftazidime and amoxiclavulanate acid, while three patients were started with a carbapenem group antibiotic plus ceftazidime or metronidazole. These patients were generally were very ill with septicaemia on presentation. Following confirmation of melioidosis through culture and/or serology, the patients were given appropriate antibiotics such as intravenous carbapenem or ceftazidime plus amoxi clavulanate or ciprofloxacin. After a month on intravenous antibiotics, they were converted to oral antibiotics consisting of amoxi clavulanate plus trimethoprim or doxycycline for an additional 6 month. Only one patient was discharged on cefuroxime and another patient with doxycycline plus ciprofloxacin.

Eleven patients had involvement of the musculoskeletal system (limbs and spine). Among these patients, one of them had splenic involvement and another three had lung involvement. Two patients with musculoskeletal melioidosis died due to overwhelming sepsis. Both of them were admitted within one week after the onset of the symptoms and presented with septic shock on admission. One had a right foot abscess and the other had pneumonia with right ankle abscess. The other nine patients improved and had no documentation of any further admissions due to a recurrence of the infection.

\section{DISCUSSION}

Meiloidosis is not uncommon in the state of Pahang. The incidence in this state is 6.1 per 100,000 population per year, similar to the rate in Northern Thailand ${ }^{3}$. We identified 11 patients with orthopaedic involvement. The true incidence of the disease is expected to be higher considering some people with milder symptoms especially those from rural areas may not have presented to the hospital ${ }^{3,10}$. Some may seek traditional medication first and/or wait for symptoms to subside. They only present to the hospital when symptoms worsen or fail to improve. The higher incidence of males with melioidosis may be due to their greater exposure to soil or contaminated water while engaging in agricultural activities ${ }^{10}$.

The majority of patients were diabetics as was the case in previous studies ${ }^{2,3,11,12}$. Other risk factors reported in literature, but not present in our patients were renal failure, HIV infection, malignancy, corticosteroid therapy, renal calculi and chronic lung disease ${ }^{3}$. There were still be 20$30 \%$ of patients with no significant risk factors ${ }^{2}$. Asymptomatic seroconversion is common in endemic countries as evidenced by positive serology reported in up to $50 \%$ of healthy adults in these countries ${ }^{1}$.

In this study, abscesses were the main cause for orthopaedic referral followed by septic arthritis and cellulitis. Abcesses accounted for 63.6 percent of the cases seen during the study period. Other studies have shown that localized meiloidosis is the most common presentation followed by meiloidosis with septicaemia ${ }^{10}$. The clinical presentation of septicaemia secondary to melioidosis is similar to sepsis caused by other organisms $s^{3,6,17}$.

Outcome of septicaemic melioidosis has improved tremendously in recent times. Mortality rates have dropped from $60 \%$ to $37 \%{ }^{3}$ in more recent studies; this is attributed to timely and appropriate empirical antibiotic therapy (ceftazidime and carbapenem). In this study, 4 out of 33 patients $(12 \%)$ died due to overwhelming sepsis This mortality rate is low when compared to another study reported in this country that quoted a $54 \%$ mortality rate ${ }^{3}$. The lower percentage of fatalities may be due to several factors: a higher index of suspicion among the medical staffs in this endemic region, adequate supportive care and the timely initiation of appropriate antibiotic therapy.

\section{CONCLUSION}

Burkholderia pseudomallei infection or melioidosis should be suspected in all patients presenting with focus of infection or sepsis especially those from endemic regions in this country. A high index of suspicion and early initiation of treatment are important to achieve a more favourable outcome and lower risk of mortality. 


\section{REFERENCES}

1. Whitmore A, Krishnaswami CS. An account of a hithero undescribed infectious disease occurring among the population of Rangoon. Indian Med Gaz 1912. 47: 262-72.

2. Currie BJ. Melioidosis: an important cause of pneumonia in residents of and travellers returned from endemic regions. Eur Respir J 2003; 22: 542-50.

3. How SH, Liam CK. Melioidosis: A Potentially Life Threatening Infection. Med J Malaysia 2006; 61(3): 386-94.

4. Currie BJ. Melioidosis: an Australian perspective of an emerging infectious disease. Recent Adv Microbiol 2000; 8: 1-75.

5. Puthucheary SD, Parasakthi N, Lee MK. Septicamic melioidosis: a review of 50 cases from Malaysia. Trans R Soc Trop Med Hyg 1992; 86(6): 683-5.

6. Pui MH, Tan AP. Musculoskletal melioidosis: clinical and imaging features. Skeletal Radiol 1995; 24(7): 499-503.

7. Tsai WC, Liu YC, Yen MY, Wang JH, Chen YS, Wang JH, et al. Septicemic melioidosis in Southern Taiwan: a case report. $J$ Microbiol Immunol Infect 1998; 31(2): 137-40.

8. Inglis TJ, Garrow SC, Adams C, Henderson M, Mayo M, Currie BJ. Acute melioidosis outbreak in Western Australia. Epidemiol Infect 1999; 123(3): 437-43.

9. Sam IC, Puthucheary SD. Melioidosis in children from Kuala Lumpur, Malaysia. Ann Trop Paediatr 2006; 26(3): 219-24.

10. How HS, Ng KH, Yeo HB, Tee HP, Shah A. Paediatric Melioidosis in Pahang, Malaysia. J Microbiol Immunol Infect 2005; 38 : 314-19.

11. Pagalavan L. Melioidosis: The Johor Bahru experience. Med J Malaysia 2005; 60 (5): 599-605.

12. Heng BH, Goh KT, Yap EH, Loh H, Yeo M. Epidemiological Surveillance of Melioidosis in Singapore. Ann Acad Med Singapore 1998; 27(4): 478-84.

13. Vuddhakul V, Tharavichitkul P, Na-Ngam N, Jitsurong S, Kunthawa B, Noimay P et al. Epidemiology of Burkholderia pseudomallei in Thailand. Am J Trop Med Hyg 1999; 60(3): 458-61.

14. Thummakul T, Wilde H, Tantawichien T. Melioidosis, an environmental and occupational hazard in Thailand. Mil Med 1999; 164(9): 658-62.

15. Romanee C, Phongsatron P, Khuanchai S, Thira S. Meliodosis at Mahraj Nakorn Chiang Mai Hospital. Thailand, J Infect Dis Antimicrob agents 2005; 22: 45-52.

16. Limmathurotsakul D, Chaowagul W, Chierakul W, Sirisanthana T. Risk factors for recurrent melioidosis in Northeast Thailand. Clin Infect Dis 2006; 43(8): 979-86.

17. Jain VK, Jain D, Kataria H, Shukla A, Arya RK, Mittal D. Melioidosis: A review of orthopaedic manifestations, clinical features, diagnosis and management. Indian J Med Sci 2007; 61(10): 580-90.

18. Kosuwon W, Saengnipanthkul S, Mahaisavariya B. Musculoskletal melioidosis. J Bone Joint Surg 1993; 75-A; 1811-5.

19. Kosuwon W, Taimglang T, Sirichativapee W, Jeeravipoolvarn P. Melioidosis septic arthritis and its risk factors. J Bone Joint Surg 2003; 85-A: 1058-61.

20. Subhadrabandhu T, Prichasuk S, Sathapatayavongs B. Localised melioidotic osteomyelitis. J Bone Joint Surg 1995; 77-B: 445-9

21. Muthusamy KA, Waran V, Puthucheary SD; Spectra of central nervous system melioidosis. J Clin Neurosci 2007; 14(12): 1213-5.

22. Nather A, David V, Hee HT, Thambiah J. Pyogenic vertebral osteomyelitis: a review of 14 cases. J Orthop Surg 2005; 13(3): $240-4$. 